Cumhuriyet International Journal of Education-CIJE

e-ISSN: 2147-1606

Vol 5 (2), 2016, $84-99$

\title{
3-5 Year Olds' Executive Function Performances and Its Relationship with Language
}

Abilities

\section{Zeynep GÜLTEKIN AHÇI}

\section{Summary}

\section{INTRODUCTION}

Executive functions are top-down cognitive processes that controls starting, sustaining, shifting and ending purposeful acts. Two widely excepted and studied primary executive components are inhibition and cognitive flexibility. Inhibition is to deliberately resist to distracters and to dominant responses while flexibility is the ability to to switch focus of attention, to shift between rules, responses, and cognitive sets, Executive functions are decisive processes on individuals' functionality in everyday life, including social, academic, and work life. Therefore, it is crucial to investigate the emergence and development of these processes early in life. Studies show that there are rapid changes in inhibition and flexibility around the ages 3 and 5. However, widely used executive function tasks for this age group yield varying results for different cultures. Thus, two popular executive function tasks for preschool children were administered to a group of 3 to 5-year-old children from families of middle socioeconomic status in Ankara, Turkey. Since those abilities are known to be closely related to language development, their correlations with receptive and expressive language abilities were investigated.

\section{METHOD}

The study was conducted in two public preschools in Ankara. Three age groups, 3, 4 and 5-year-olds, were included in the study. Seventy children (34 girls and 36 boys) were tested. Instruments used in data collection were Day/Night task (DNT), Flexible Item Selection Task (FIST) and Turkish Expressive and Receptive Language Test (TIFALDI). The data were non-normally distributed and for intergroup comparisons Kruskal-Wallis and Mann-Whitney U tests were conducted. As to the tests of correlation, Spearman Rho was adopted.

\section{FINDINGS}

The results showed that the performance in FIST increased with age. Threeyear-olds' first correct selection performances, which requires abstraction, were significantly lower than 4- and 5-year-olds' performances. There was no significant difference between 4- and 5-year-olds. The second correct selection, which requires both abstraction and cognitive flexibility, showed that 5-year-olds did significantly better than 3-year-olds, but did not differ from 4-year-olds significantly. Besides, first selection or abstraction performances had significant relationship with receptive language skill at age 4 . On the other hand, DNT correct response performances revealed no sgnificant age differences and no significant correlations with TIFALDI. 


\section{DISCUSSION}

There were no significant age related differences for the inhibition task DNT. This result may be accounted for cultural differences. Yet there were wide individual differences across all ages. Therefore in order to draw conclusions on the task and the development of inhibition in this age group require more research.

Cognitive flexibility task FIST was found to be sensitive to developmental changes in this age group. The results showed that abstraction was acquired solidly at the age of 4 . However, cognitive flexibility emerged around age 5 and far from fully developed.

Only FIST abstraction performance yielded a significant correlation with TIFALDI receptive language performances at age 4 . The rest of the correlations between flexibility, abstraction and language performances were found to be the common effect of age. Based on these results, it is possible that age 4 is the intersection point of abstraction ability and receptive language. On the other hand, it is also possible that since abstraction performance reached the ceiling at age 4 , the correlation lost its significance at age 5 .

\section{SUGGESTIONS}

Additional research is needed to evaluate the tasks adopted in the study. Further studies with diverse tasks on wider age range and different sociocultural groups would provide more comprehensive and definitive information about both tasks and development of those abilities. 